\title{
LoopTag FRET Probe System for Multiplex qPCR Detection of Borrelia Species
}

\author{
Henning Hanschmann ${ }^{1, \dagger}{ }^{\dagger}$ Stefan Rödiger ${ }^{2,3, *,+} \mathbb{D}$, Toni Kramer ${ }^{1}$, Katrin Hanschmann ${ }^{1, \dagger}$, Michael Steidle ${ }^{4, \dagger}$, \\ Volker Fingerle $^{5,+}+\mathbb{D}$, Carsten Schmidt ${ }^{2,+}+\mathbb{D}$, Werner Lehmann ${ }^{1,+}$ and Peter Schierack ${ }^{2,3, *}$ \\ 1 Attomol GmbH, 03205 Bronkow, Germany; h.hanschmann@attomol.de (H.H.); tonikramer90@aol.de (T.K.); \\ k.hanschmann@attomol.de (K.H.); w.lehmann@attomol.de (W.L.) \\ 2 Institute of Biotechnology, Faculty of Nature and Science, Brandenburg University of Technology \\ Cottbus-Senftenberg, 01968 Senftenberg, Germany; ycschmidty@gmail.com \\ 3 Faculty of Health Brandenburg, Brandenburg University of Technology Cottbus-Senftenberg, \\ 03046 Senftenberg, Germany \\ 4 Laborärzte Sindelfingen, 71065 Sindelfingen, Germany; m.steidle@laboraerzte-sifi.de \\ 5 German National Reference Center for Borrelia, 85764 Oberschleißheim, Germany; \\ Volker.Fingerle@lgl.bayern.de \\ * Correspondence: stefan.roediger@b-tu.de (S.R.); peter.schierack@b-tu.de (P.S.) \\ + These authors contributed equally to this work.
}

check for updates

Citation: Hannschmann, H.; Rödiger, S.; Kramer, T.; Hanschmann, K.; Steidle, M.; Fingerle, V.; Schmidt, C.; Lehmann, W.; Schierack, P. LoopTag FRET Probe System for Multiplex qPCR Detection of Borrelia Species. Life 2021, 11, 1163. https://doi.org/ $10.3390 /$ life11111163

Academic Editor: Armen Mulkidjanian

Received: 21 September 2021

Accepted: 28 October 2021

Published: 31 October 2021

Publisher's Note: MDPI stays neutral with regard to jurisdictional claims in published maps and institutional affiliations.

Copyright: (c) 2021 by the authors. Licensee MDPI, Basel, Switzerland. This article is an open access article distributed under the terms and conditions of the Creative Commons Attribution (CC BY) license (https:/ / creativecommons.org/licenses/by/ $4.0 /)$.

\begin{abstract}
Background: Laboratory diagnosis of Lyme borreliosis refers to some methods with known limitations. Molecular diagnostics using specific nucleic acid probes may overcome some of these limitations. Methods: We describe the novel reporter fluorescence real-time polymerase chain reaction (PCR) probe system LoopTag for detection of Borrelia species. Advantages of the LoopTag system include having cheap conventional fluorescence dyes, easy primer design, no restrictions for PCR product lengths, robustness, high sequence specificity, applicability for multiplex real-time PCRs, melting curve analysis (single nucleotide polymorphism analysis) over a large temperature range, high sensitivity, and easy adaptation of conventional PCRs. Results: Using the LoopTag probe system we were able to detect all nine tested European species belonging to the Borrelia burgdorferi (sensu lato) complex and differentiated them from relapsing fever Borrelia species. As few as 10 copies of Borrelia in one PCR reaction were detectable. Conclusion: We established a novel multiplex probe real-time PCR system, designated LoopTag, that is simple, robust, and incorporates melting curve analysis for the detection and in the differentiation of European species belonging to the Borrelia burgdorferi s.l. complex.
\end{abstract}

Keywords: Borrelia; B. Burgdorferi (sensu lato) complex; real-time PCR; Lyme borreliosis; diagnostic

\section{Introduction}

Lyme borreliosis is a multi-system disorder caused by several species of the Borrelia burgdorferi (sensu lato) (s.l.) complex. Only in Europe, it is estimated that there are more than 200,000 cases per year, although the number of unreported cases is very likely higher. Borrelia are transmitted to humans by bites of infected ticks. The disease primarily affects the skin, joints, and the nervous system [1-6]. In Europe the genospecies B. burgdorferi sensu stricto (s.s.), B. afzelii, B. bavariensis, B. garinii, B. lusitaniae, B. spielmanii are assured to be human pathogenic while for the other detected species, namely $B$. bissettii, $B$. valaisiana, and B. kurtenbachii human pathogenicity is unclear. However, the most prevalent species in Europe are B. garinii, B. afzelii, B. burgdorferi s.s., and B. valaisiana [4,7].

The diagnosis of Lyme borreliosis is based on medical patient history and clinical symptoms. Microbiological analyses are usually based on the indirect detection of B. burgdorferi s.l. infection by antibody detection using an enzyme-bound immunosorbent assay (ELISA). Although the ELISA method is widely used, this method has technical limitations due to the assay principle [8] and biological limitations by delaying antibody 
formation, high dependence on the stage and disease manifestations, cross-reactivity, and high seroprevalence in healthy populations in endemic areas. The later makes it difficult to detect a re-infection by using such type of test. The cultivation of Borrelia spirochete is not applied in clinical practice. This is due to long and challenging cultivation, poor sensitivity, and susceptibility to impurities $[9,10]$.

Besides serological testing, quantitative polymerase chain reactions (qPCRs) are widely used detection methods for diagnostic and research purposes [4,7,9-11] since PCRs are faster, easier and in many instances more sensitive than cultures [12]. Advantages of qPCR include quantification and differentiation. The target DNA amount in a sample can be determined by observing the change in fluorescence as a function of the number of PCR cycles. From this, the $\mathrm{Cq}$ value (quantification cycle) is calculated using various methods. High Cq values are characteristic for samples with little target DNA in the sample. Further details are described in [13]. There are fundamentally two methods of distinguishing the target DNA. These are, on the one hand, probe-based detection, wherein a target DNA is detected in a sequence-specific manner due to the molecular interactions. On the other hand, a target DNA can be distinguished from other sequences due to its melting temperature $(\mathrm{Tm})$. This presupposes that the melting temperature and constant reaction conditions are determined. These basic approaches can also be combined, as described in the following sections. Quantitative diagnostic data are relevant for establishing correlations between Borrelia burden and patient symptoms [12,14].

The potential of the PCR for sensitive and fast nucleic acid detection became obvious immediately after its publication by Saiki et al. in 1986 [15] and was soon adapted in laboratory diagnosis of human Borrelia [16]. The PCR is a non-quantitative endpoint reaction and is suitable for sensitive detection of DNA. Shortly after the development of the real-time PCR method with which quantitative detection of target DNA molecules is possible in realtime. Basically, the kinetics of the PCR reaction is observed and evaluated in real time. An important milestone for the usability of this real-time PCR technology was the introduction of specific TaqMan probes and intercalating dyes $[17,18]$. These probes increased the specificity and accuracy of quantitative assays and reduced the risk of cross-contamination compared to conventional PCRs $[13,19]$. Though qPCR is very sensitive, the detection limit is mainly negatively affected by sampling (leads to a low amount of input DNA), extraction (loss of sample DNA due to the extraction), and reverse transcription for samples starting with RNA instead of DNA [20]. qPCRs are routinely used for numerous applications like gene expression analysis, genotyping, and pathogen detection [13,21,22].

Several probe and primer systems using quenching processes (e.g., Scorpions, Molecular Beacons, or Ampliflour primer) or Foerster Resonance Energy Transfer (FRET, e.g., hybridization probes) for signal generation [23-25] have been developed. Probe systems are also applied in other assay platforms such as microarrays and microbead assays [26]. FRET is a physical phenomenon relying on the proximal distance-dependent radiation-less transfer of energy from an excited donor molecule, that initially absorbs energy, to an acceptor dye molecule leading to a measurable increase in emission at the acceptor dyespecific wavelength. This transfer results in a wavelength shift between excitation and emission. FRET partners basically come into close spatial relation when two different probes hybridize in close spatial proximity. The upstream binding probe has a dye-labeled $3^{\prime}$-end, and the downstream binding probe a dye-labeled 5 '-end, with both dyes forming an active FRET pair only when they bind to a common target. The FRET detection signal increases in direct proportion to the formation of specific homogeneous active FRET pairs binding to targets and allows for reliable readouts in assays [27].

An advantage of FRET systems lies in their use for melting curve analysis. Here, hybridized double-stranded DNA bound with dye is heated until its melting point (Tm), where a sudden decrease in measured fluorescence occurs due to dissociation and release of the dyes. If the Tms of several hybridized DNA sequences differ, then multiplex PCRs can be designed: the presence of different targets can be analyzed by melting curve analyzes, although the same FRET pair is used to visualize the amplification [28]. A disadvantage 
of classical hybridization probe systems is the need for two labeled probes for the detection of one target, which may reduce sensitivity due to a higher number of included oligonucleotides [29]. This factor is an important consideration in designing multiplex PCRs. The reduction in the number of oligonucleotides does not only reduce complexity of the reaction but also costs for consumables. We established a novel multiplex probe real-time PCR system, designated LoopTag [30], that is simple, robust, and incorporates melting curve analysis for the detection and differentiation of European species belonging to the B. burgdorferi s.l. complex.

\section{Materials and Methods}

\section{1. qPCR Reaction and Melting Curve Analysis}

Real-time PCRs were performed with a LightCycler® 1.5 and a LightCycler ${ }^{\circledR} 2.0$ (Roche, Germany) using LightCycler® FastStart DNA Master HybProbe Kit (Roche, Germany). The PCR program encompasses $95^{\circ} \mathrm{C} / 7 \mathrm{~min}$ initial denaturation followed by 45 cycles comprising $95^{\circ} \mathrm{C} / 4 \mathrm{~s}$ denaturation, $62^{\circ} \mathrm{C} / 25 \mathrm{~s}$ annealing, and $72{ }^{\circ} \mathrm{C} / 15 \mathrm{~s}$ elongation. Afterward, a melting curve analysis was performed with $95^{\circ} \mathrm{C} / 3 \mathrm{~s}, 50^{\circ} \mathrm{C} / 10 \mathrm{~s}, 40^{\circ} \mathrm{C} / 20 \mathrm{~s}$ followed by a constant increase of $0.2{ }^{\circ} \mathrm{C} / \mathrm{s}$ until $85^{\circ} \mathrm{C}$. The amplification was monitored during the annealing phase. The design of primers and probes was done following principles described in [31]. The formation of secondary structures and oligonucleotide dimers was studied with Mfold [32] and PerlPrimer [33], respectively. Primer and dye-labeled probes were purchased from Biotez (Germany) and IBA (Germany). The forward primer had the sequence $5^{\prime}$ ATG GAG CCG CAA TCA TTG CCA TTG CAG A 3' (GC: 50\%, $28 \mathrm{nt}$, Tm in $_{\text {ilico }}$ : $71.67^{\circ} \mathrm{C}$ ) which included the target-unspecific $5^{\prime}$-sequence (bold), and the Borrelia-specific primer sequence as published by Schwaiger et al. (2001) [29]. At its $5^{\prime}$-end it was labeled with fluorescein isothiocyanate (FITC, Emission: $510 \mathrm{~nm}$ ). The reverse primer was nonlabeled and had the Borrelia-specific sequence $5^{\prime}$ AGC AAA TTT AGG TGC TTT CCA A3 ${ }^{\prime}$ (GC: $36 \%, 22 \mathrm{nt}, \mathrm{Tm}_{\text {in silico }}: 59.54{ }^{\circ} \mathrm{C}$ ) as described by Schwaiger et al. (2001) [29].

The primer pair amplified a sequence of the flagellin gene with a size of 180 base pairs. The internal amplification control was synthesized by Biotez and had the sequence $5^{\prime}$ GCA ATC ATT GCC ATT GCA GAG GCG GTT TGC GTA TTG GGC GCC AGG GTG GTT TTT CTT TTC ACC AGC GAG ACG GGC AAC AGC TGA TTG CCC TTC ACC GCC TGG CCC TGA GAG AGT TGC AGC AAG CGG TCC ACG CTG GTT GGA AAG CAC CTA AAT TTG $C 3^{\prime}$. This probe design results in different melting temperatures for each gene (Table 1). The amplification control was amplified with the Borrelia-specific primer pair. For the detection and differentiation of Borrelia species a probe with the sequence $5^{\prime} \mathrm{CAA}$ TGA CAG ATG AGG TTG TAG CAG CAA CAA CTA ATA GTA GTG GCT CCA T $3^{\prime}$ was designed based on the alignment of the Borrelia flagellin gene. At its $3^{\prime}$-end it was labeled with Atto 590 (emission: $640 \mathrm{~nm}$ ). The probe for the detection of the amplification control had the sequence $5^{\prime}$ TGA AAA GAA AAA CCA CCC TGG CGC CCA AAG TGG CTC CAT $3^{\prime}$ and was $3^{\prime}$-labeled with Cy5.5 (emission: $705 \mathrm{~nm}$ ). Color compensation was performed according to the manufacturer's recommendations.

\subsection{Borrelia Strains and DNA Extraction and $q P C R$ Conditions}

Borrelia species and strains are listed in Table 2. Genomic DNA was isolated using QIAamp DNA Mini Kit (Qiagen Corporation, Hilden, Germany). Genomic DNA was stored frozen in $\mathrm{ddH}_{2} \mathrm{O}$ until further use. For PCR, $5 \mu \mathrm{L}$ template was mixed with $5 \mu \mathrm{L}$ reaction mixture $\left(8 \mathrm{mM} \mathrm{MgCl} 2,0.4 \mu \mathrm{M}\right.$ primer and probes, $8.24 \times 10^{-12} \mu \mathrm{M}$ internal amplification control, $2 \times$ FastStart reaction mix). For determination of the detection limits (LoD) a dilution series ranging from $1 \mathrm{pg}$ to $0.1 \mathrm{fg}$ DNA per reaction was performed. The samples were provided semi-blinded by the German National Reference Center for Borrelia (Germany). Samples with unknown quantities of samples were provided for this purpose and were scanned using the LoopTag system. The Cq values and melting points determined by LoopTag-PCR were finally compared with the sample amounts. DNA concentrations were quantified with a NanoDrop instrument (Thermo Fisher, Waltham, 
MA, USA). At this point, it should be noted that the NanoDrop is not always the best nucleic acid quantification method [34-36]. An evaluation of further methods is recommended. The DNA was diluted in $\mathrm{ddH}_{2} \mathrm{O}$. For comparing the LoopTag system with the intercalating dye EvaGreen [21,37] (Biotium, Fremont, CA, USA) one sample (B. lusitaniae Poti B3, $60 \mathrm{ng} / \mu \mathrm{L}$ ) was diluted in water up to $1: 10^{7}$, and the same primer pair was used. PCR tests were performed in triplicates for each dilution. After each PCR reaction melting curves were analyzed.

Data Analysis

Amplification data and melting curve data were exported from the LightCycler as comma separated values. RKWard [38] (v. 0.7.2) and dedicated $R$ packages were used for all analysis as described here [39]. The report package (v. 0.3.0) [40] was used for report generation. In detail, amplification curves were preprocessed with functions from the chipPCR package [41] (v. 1.0.2). The RFU values are the ratio of the 640/530 channel. The $\mathrm{Cq}$ values were calculated by the scale-insensitive marker second-derivative maximum (SDM) [42]. The amplification efficiency was determined with the effcalc function from the chipPCR package using a decadic dilution series. Melting curves were analyzed using the MBmca package [43] (v. 1.0.1.1) as described in [39].

\section{Results}

\subsection{Mechanism of the LoopTag System}

The principle of the LoopTag real-time PCR probe system is shown in Figure 1. The forward primer hybridizes onto a target $3^{\prime}-5^{\prime}$ DNA strand leading to elongation during PCR. Denaturation of the newly formed strand leads to a new $5^{\prime}-3^{\prime}$ strand with the attached primer plus acceptor dye. The binding of the probe and reverse primer hybridization leads to looping allowing the transmission of energy from a donor molecule to an acceptor molecule. The transfer of energy leads to a reduction in the donor's fluorescence intensity and consequently its excited state lifetime, and to a corresponding measurable increase in the acceptor's emission intensity that can be monitored during real-time PCR amplification. Reverse primer elongation then occurs leading to the formation of a new DNA strand (Figure 1).

\subsection{Amplification of the Flagellin Gene}

An amplification of the flagellin gene of the following species and strains of the B. burgdorferi s.l. complex was detected: B. valaisiana strain VS116; B. lusitaniae strains Poti B2 and Poti B3; B. burgdorferi s.s. strains B31, PKa2, and PBre; B. spielmanii strain PSig2; B. bavariensis strain PBi; B. garinii strains PLa PBr, PHei, TN, PRef, and PWudII; B. afzelii strains PKo, PGau, and PVPM; B. bissettii strain PGeb; B. kurtenbachii strain 25015. Regarding the relapsing fever group Borreliae, the species B. miyamotoi and B. recurrentis were not detectable while $B$. parkerii, B. anserina, B. duttonii, and B. turicatae were detectable at very high template concentration per reaction (more than $0.64 \mathrm{ng}$ or $0.32 \mathrm{ng}$ for $B$. turicatae, data not shown). Results of the amplification curves of several strains are shown in Figure 2A. Our system did not amplify the flagellin gene of related species like two Treponema phagedenis strains and two pathogenic Leptospira strains as well as two Escherichia coli (E. coli) isolates. 


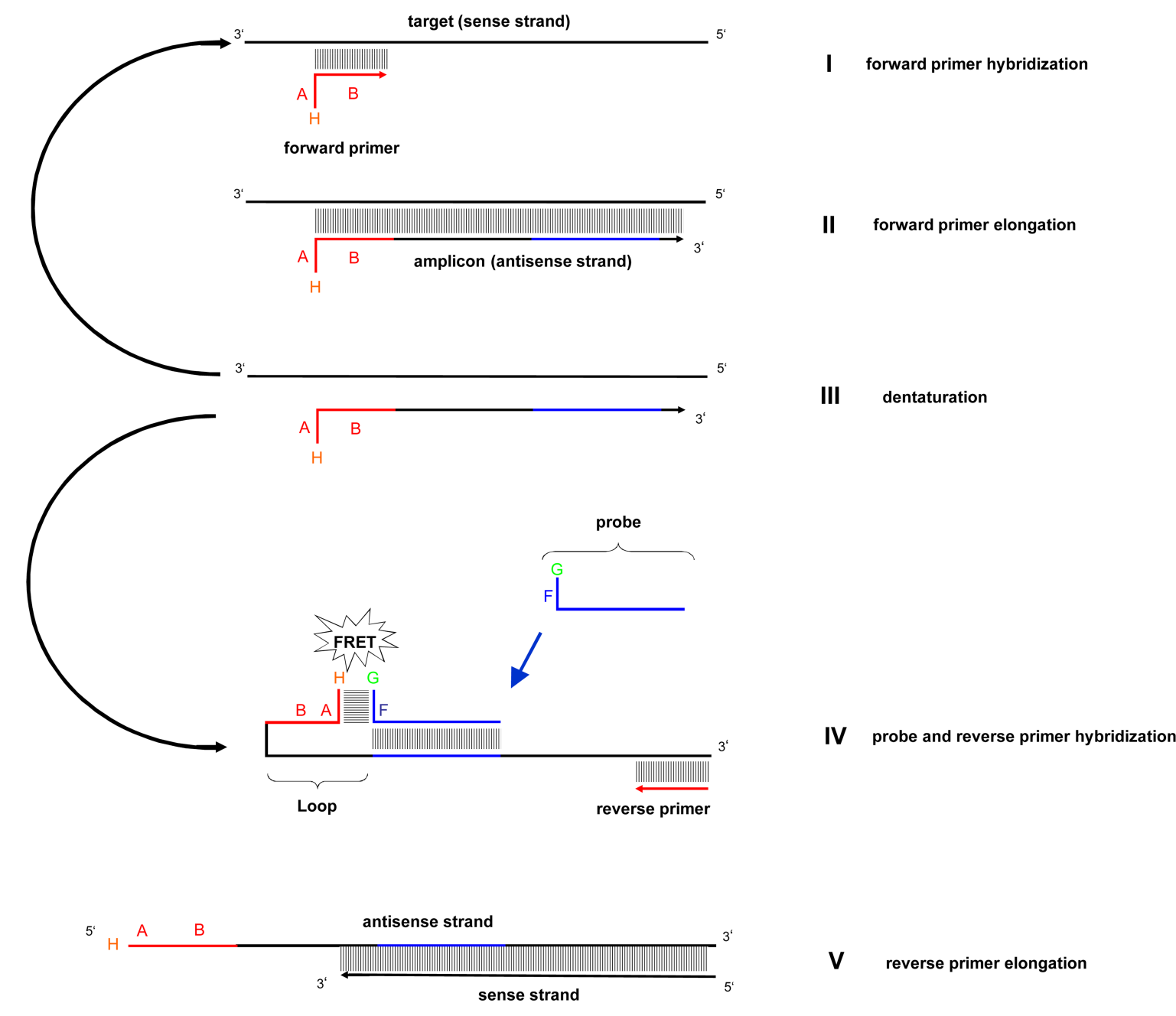

Figure 1. The diagram shows the successive stages of the LoopTag system during PCR. Please note the different reactions of the target, and the amplicon strand respectively. The mechanism is explained in the text. The forward primer of the Borrelia flagellin primer pair carries an 8 base pair long target-unspecific $5^{\prime}$-sequence (A), and a 20 base pair long targetspecific sequence (B) which hybridizes to the target (stage I). The forward primer is elongated by the polymerase (stage II). After denaturation (stage III) the probe hybridizes to the antisense strand. The target-unspecific $5^{\prime}$-sequence of the antisense strand (A) is designed to form a loop by hybridization to its complementary sequence, which is the target-unspecific part (F) of the probe. The loop brings together the fluorescence donor $(\mathrm{G})$ and the fluorescence acceptor (H), both covalently attached to the probe and the forward primer, respectively. This results in a FRET signal. The FRET signal is proportional to the number of amplification products. The reverse primer of the Borrelia flagellin primer pair hybridizes to the antisense strand (stage IV) and is elongated by the polymerase (stage V). After stage 5, the circle continues.

\subsection{Differentiation of Borrelia Species Based on Melting Curve Analysis}

The LoopTag system was established for the multiplex differentiation of pathogens. With one primer pair (low PCR complexity) the respective target is amplified, quantified, and defined by the melting temperature (Tm) of the PCR product. As shown in Figure 2B each PCR product of a specific flagellin sequence had a defined melting temperature. PCR products of all strains of one species had the same melting temperature (Table 1, Appendix A). 

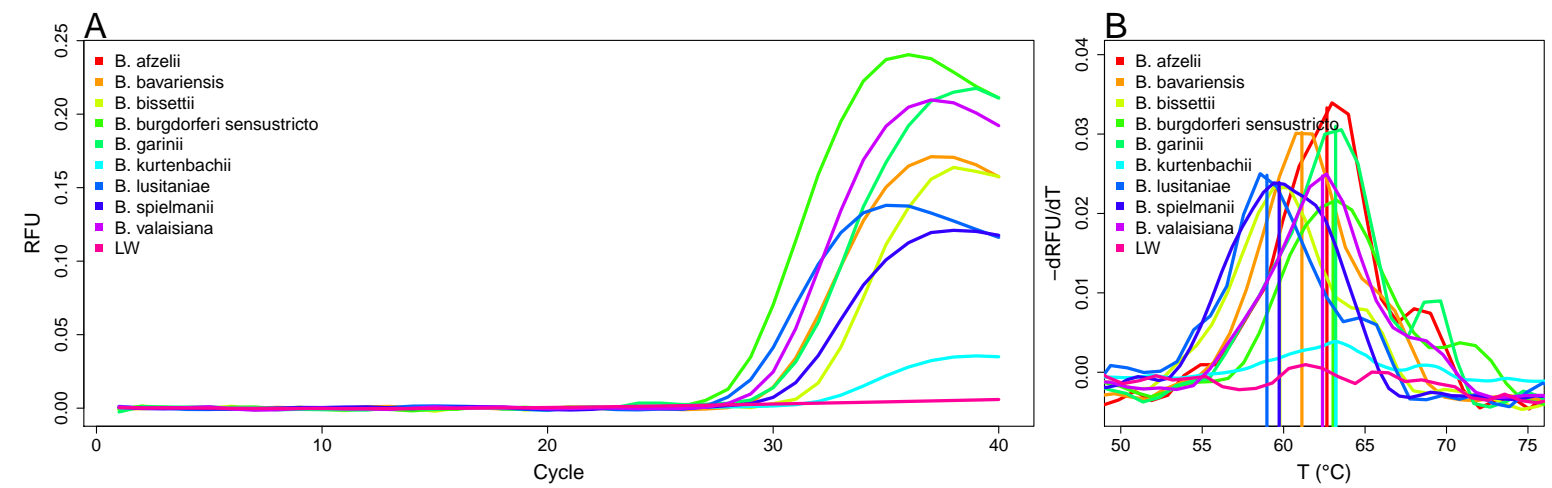

Figure 2. Amplification of the flagellin gene. (A) Plots of the amplification curves for the detection of the flagellin gene of various Borrelia. Flagellin gene of: B. afzelii PKo, B. bavariensis PBi, B. bissettii PGeb, B. burgdorferi s.s. B31, B. garinii PLa, B. kurtenbachii 25015, B. lusitaniae Poti B2, B. spielmanii PSig2, and B. valaisiana VS116. (B) Melting peak analysis. Single melting peaks of the Borrelia species: B. afzelii PKo $\left(62.4 \pm 0.1^{\circ} \mathrm{C}\right)$, B. bavariensis $\mathrm{PBi}\left(61.1 \pm 0.4^{\circ} \mathrm{C}\right)$, B. bissettii PGeb $\left(60.2 \pm 0.8^{\circ} \mathrm{C}\right)$, B. burgdorferi s.s. B31 $\left(63.7 \pm 0.7^{\circ} \mathrm{C}\right)$, B. garinii PLa $\left(62.3 \pm 0.3^{\circ} \mathrm{C}\right)$, B. kurtenbachii $25015\left(63.6 \pm 0.3^{\circ} \mathrm{C}\right)$, B. lusitaniae Poti B2 $\left(59.7 \pm 1.0^{\circ} \mathrm{C}\right)$, B. spielmanii PSig2 $\left(59.1 \pm 0.5^{\circ} \mathrm{C}\right)$, and B. valaisiana VS116 $\left(62.4 \pm 0.2{ }^{\circ} \mathrm{C}\right)$. A nontemplate water control (LW) exhibited no melting peak. Melting temperatures were calculated on the basis of three independent experiments.

Table 1. Tms and their standard deviations per species. The melting points and the standard deviations were determined by means of 5-6 measurements.

\begin{tabular}{lc}
\hline Species & Melting Temperature $\left({ }^{\circ} \mathbf{C}\right)$ \\
\hline B. afzelii & $62.4 \pm 0.1$ \\
B. bavariensis & $61.1 \pm 0.4$ \\
B. bissettii & $60.2 \pm 0.8$ \\
B. burgdorferii & $63.7 \pm 0.7$ \\
B. garinii & $62.3 \pm 0.3$ \\
B. kurtenbachii & $63.6 \pm 0.3$ \\
B. lusitaniae & $59.7 \pm 1$ \\
B. spielmanii & $59.1 \pm 0.5$ \\
B. valaisiana & $62.4 \pm 0.2$ \\
\hline
\end{tabular}

The melting temperature is an affinity measure of the probe to the target sequence. Therefore, we analyzed the relationship between the probe affinity per species (melting temperature) on the plateau height. Since the melting temperatures between the species may differ greatly, we studied how the probe affinity per species affects the plateau height. For minimizing the influence of noise in amplification curves, not the maximum but the 99th percentile of the amplification curve was used as the plateau value (Figure 3). The Pearson's product-moment correlation between the species-specific melting temperature and plateau height is significant, large, and positive $(\mathrm{r}(7)=0.94,95 \% \mathrm{CI}(0.74,0.99), p<0.001)$.

\subsection{Internal Control for the Detection and Differentiation of Borrelia Species}

An internal control is a prerequisite for specific pathogen detection in clinical samples. We established a control system based on an artificial DNA sequence, which was framed by sequences complementary to the Borrelia flagellin primer. Thus, the internal control sequence was amplified with the same primers like the Borrelia target sequences. The probe of the internal control, bound to the artificial DNA sequence, was labeled with Cyanine 5.5 (Cy5.5). The Borrelia-specific probe was labeled with the fluorescence dye Atto 590 (AttoTec, Siegen, Germany). Conclusively, amplification of the internal control was quantifiable in a second non-interfering fluorescence channel. 


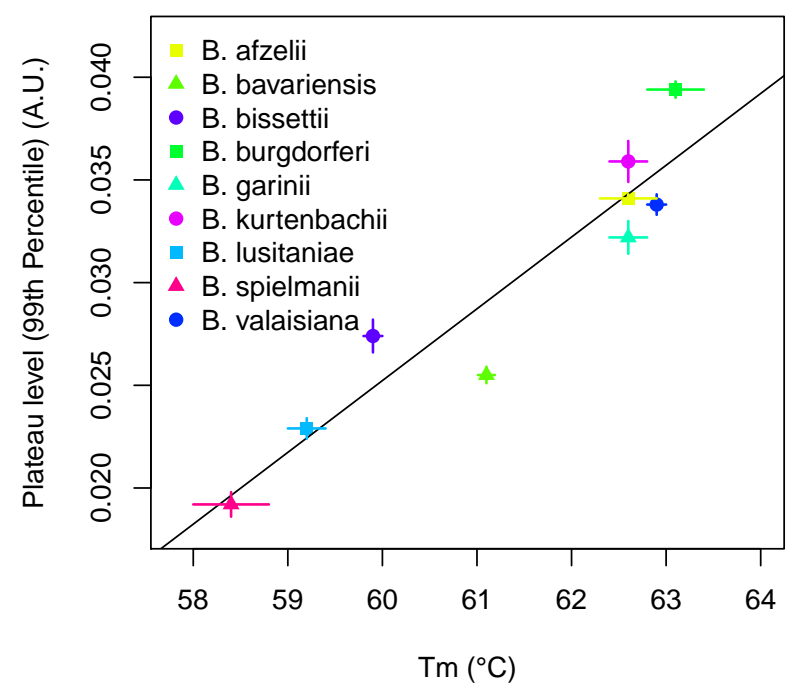

Figure 3. Probe affinity per species affects the plateau height.

\subsection{Sensitivity and Efficiency}

The comparison of the LoopTag system to a system using EvaGreen detection (as intercalating dye) revealed a similar efficiency and sensitivity (Figure 4). The minimal detectable amount of genome equivalents per strain is listed in Table 2.

Table 2. Minimal detectable amount of genome equivalents per species. DMAGE, Detectable minimal amount of genome equivalents per PCR reaction. ${ }^{*}$, data are based on determinations of in vitro cultivated Borrelia species. ${ }^{\S}$, different strains of one species. Serotypes: PHei, TN, PRef, PLa, PWudII.

\begin{tabular}{lll}
\hline Borrelia & Species & DMAGE \\
\hline & B. afzelii & $\geq 10$ \\
& B. bavariensis & $\geq 10$ \\
& B. bissettii & $\geq 10$ \\
& B. burgdorferi s.s. & $\geq 10$ \\
\cline { 2 - 3 } & & PBr : approx. 10 \\
& & PHei: $\geq 10$ \\
Borrelia burgdorferi s.l. complex & B. garinii & TN: approx. 700 \\
& & PRef: $\geq 10$ \\
& & PLa: $\geq 10$ \\
& B. kurtenbachii & PWudII: $\geq 10$ \\
& B. lusitaniae & 100 \\
& B. spielmanii & $\geq 10$ \\
& B. valaisiana & $\geq 10$ \\
& B. anserina & $\geq 10$ \\
\hline B. duttonii & $\geq 400.000 *$ \\
& B. miyamotoi & $\geq 400.000^{*}$ \\
& B. parkerii & Not detectable \\
& B. recurrentis & $\geq 400.000 *$ \\
B. turicatae & Not detectable \\
& E. coli (2 strains) & $\geq 200.000 *$ \\
\hline & Leptospira (2 strains) & Not detectable \\
& Treponema phagedenis (2 strains) & Not detectable \\
& &
\end{tabular}



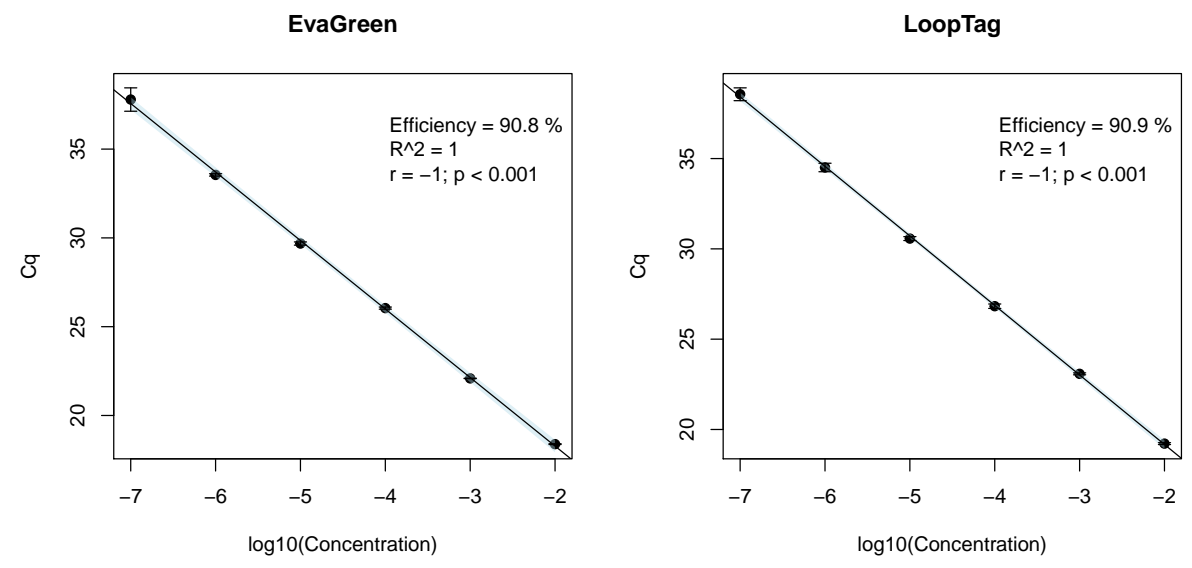

Figure 4. Comparison of the LoopTag system to a system using the common intercalating dye EvaGreen for detection. We found no pertinent differences between the LoopTag and EvaGreen detection systems. The amplification efficiency was approximately $91 \%$.

\section{Discussion}

Laboratory Borrelia diagnosis refers to some methods with known limitations. Antibody assays suffer from low sensitivity during an early stage of disease or from lower specificity caused by cross-reactions. Moreover, differentiation between an active infection and antibodies from former infections is difficult as antibodies persist after an infection has been overcome: in the normal population the prevalence of antibodies to B. burgdorferi is up to $25 \%$, depending on age and gender. Additionally, detection by culture, combined with microscopic detection or serological testing, is in use [44]. However, the method is time-consuming, needs special equipment, special experience, and the sensitivity is low, ranging from $40-70 \%$ for skin biopsy specimens, and $10-30 \%$ for cerebrospinal fluid. Although false-positive or false-negative results occur, PCR is the most modern technique for detecting human pathogenic Borrelia burgdorferi (sensu lato) complex species $[9,44,45]$. PCR tests overcome some limitations of current diagnostic techniques and are valuable additional tools for the diagnosis of Lyme borreliosis. Real-time quantitive PCR has sped up the molecular diagnosis of pathogens during the last decade. This process was impelled essentially by the development of different molecular probe systems.

Here, we describe a probe system designed for a multiplex quantitative PCR test for the detection and differentiation of Borrelia species. In comparison to other probe systems, the most important advantage of this system is that only a single probe with a simple $3^{\prime}$ end fluorescent marking is required for the melt curve analysis. The second label, e.g., a standard fluorescein label, is conjugated to the $5^{\prime}$-end of one primer. This is advantageous for multiplexing as this approach reduces costs, and the number of oligonucleotides per reaction. The degree of multiplexing depends on the sensor technology of the measuring instrument, the available FRET pairs, the probe regions (differences in Tms), and the PCR conditions (e.g., salt concentration, $\mathrm{pH}$ value). We focused on a multiplex level that is sufficient for this diagnostic application.

Loop formation may initiate complex secondary DNA structures, since loops may also hybridize internally within minimally complementary regions. However, loop or hairpin formation is a molecular process often applied in probe systems [21,24]. In this respect, our LoopTag system is similar to Molecular Beacons, which also form a stem-loop-like structure only upon hybridization to a target. This approach allows for a multiplexed melting point analyzes [21,28]. The properties of the LoopTag system are that fewer probes/primers are in the mix, that the position of the detection probes can be flexibly selected and that the stem sequences are suitable for other target detections.

Loop formation of the LoopTag system between one of the primers, and the probe appears not to inhibit amplification. In contrast to other FRET probe systems reported [21], the LoopTag system shows large variability about the binding site of the probe. Nonetheless, 
the loop structure is crucial for melting curve analysis, which demands a careful amplicon design. The shape of the amplification curves regularly shows a hook-like shape, as is also known from other probe systems [46]. Each standard PCR, independent of the amplicon length, could be adjusted by adding the stem sequences to one primer and by designing the probe. This also applies to multiplex PCRs.

The LoopTag system utilizes sequence-specific probes in combination with the primers. It is well accepted that this combination has a higher specificity than intercalating dyes. The specificity of the applied test system is indeed very good. All included European species of the B. burgdorferi s.l complex were detectable. Relapsing fever Borrelia were not detectable or only at unphysiological high DNA concentrations. Small differences in the gene sequence of the flagellin gene within the species of the B. burgdorferi s.l complex results in different Tms. However, the high sequence homology of the flagellin gene and the standard deviations of the Tms makes it difficult to distinguish each species from another by several degrees Celsius. This is a natural limit for the multiplexing degree. Each system has defined technical limits, such as serological tests (see introduction). We have tested known strains and therefore cannot study the melting temperatures of all existing strains. Whenever the sequences have a high similarity, some strains will have similar melting temperatures. For example, B. lusitaniae and B. bissettii are similar in their melting temperature. The pathogenicity of both strains has not yet been fully clarified and they do not occur frequently. In an unknown sample with a melting temperature (e.g., $60{ }^{\circ} \mathrm{C}$ ), pathogens would be detected due to the primer specificity but would not be differentiated. In such cases, information, such as epidemiological data, should be taken into account more strongly. Further analyses (e.g., sequencing, further LoopTag probe system) could also be carried out. Thus, our probe system can be used for the detection of borrelia to distinguish B. burgdorferi s.l. strains in the delimitation of relapse fever strains. The latter are detected by the present system in physiological concentrations. The differentiation between B. burgdorferi s.l. strains is possible with restrictions. Although individual species have very similar melting temperatures, it is possible to associate them with groups having the same melting temperature. The LoopTag system possesses a large linear detection range of at least between 10 and 1,000,000 copies per sample. Our system has not yet been tested in multiple global laboratories and therefore does not meet the requirements for a companion diagnostic (CDx). However, there are other application scenarios for our probe system. On the one hand, it can be used in clinical research to screen samples. On the other hand, the system can be used in routine clinical practice as a laboratory-developed test (LDT, use within a single laboratory), as we have disclosed all sequences in this study.

Another application is the use of the LoopTag system in combination with planar array technologies indicating its versatility. In a proof-of-concept study we transferred the LoopTag system for the multiplex detection of PCR products on the surface of microbeads for a real-time monitoring and surface melting curve analysis (see Appendix B).

\section{Conclusions}

We designed a new probe system, called LoopTag, for the detection and the differentiation of PCR products. In our study we applied the technology on Borrelia species. The system is simple and offers the ability to perform melting curve analysis. We validated the LoopTag system for the detection and differentiation of European species of the B. burgdorferi s.l. complex and the distinguishing from relapsing fever Borrelia species. We show a high specificity. We also show a high sensitivity down to 10 genome equivalents per PCR reaction.

\section{Patents}

The LoopTag system is patented [30].

Author Contributions: Conceptualization, W.L. and H.H.; methodology, W.L. and H.H.; software, S.R.; validation, T.K., H.H. and K.H.; formal analysis, H.H. and S.R.; investigation, C.S., H.H., T.K. and S.R.; resources, V.F., W.L. and M.S.; data curation, S.R., H.H. and T.K.; writing-original draft 
preparation, H.H., P.S., W.L. and S.R.; writing-review and editing, all authors; visualization, W.L., H.H. and S.R.; supervision, W.L., S.R. and P.S.; funding acquisition, W.L. and P.S. All authors have read and agreed to the published version of the manuscript.

Funding: This work was supported by InnoProfile 03IP611 funded by the Federal Ministry of Education and Research (BMBF, Bonn, Germany) and by ZIM KF2212801SK9 funded by the Federal Ministry for Economic Affairs and Energy (BMWi, Berlin, Germany).

Institutional Review Board Statement: Not applicable.

Informed Consent Statement: Not applicable.

Data Availability Statement: The data for this study are stored on https: / codeberg.org/devSJR/ LoopTag_data (accessed on 27 October 2021). This includes the raw data (file formats: txt, xlsx) of the amplification curves and melting curves from Roche's LightCycler systems, and the VideoScan platform. All data were published under the GPL-3 or later license for research purposes.

Conflicts of Interest: Werner Lehmann has a management role and is a shareholder of Attomol $\mathrm{GmbH}$. Henning Hanschmann and Katrin Hanschmann are employees of Attomol GmbH. This company is a diagnostic manufacturer. All other authors declare that they have no competing financial interests.

\section{Abbreviations}

The following abbreviations are used in this manuscript:

$\begin{array}{ll}\text { A.U. } & \text { Arbitrary units } \\ \text { BHQ2 } & \text { Black Hole Quencher 2 } \\ \text { Cq } & \text { Cycle of quantification by SDM method } \\ \text { est } A 1 & \text { Heat-stable toxin I gene } \\ \text { FRET } & \text { Foerster Resonance Energy Transfer } \\ \text { qPCR } & \text { Quantitative PCR } \\ \text { RFU } & \text { Relative fluorescence units [Arbitrary Unit] } \\ \text { rMFI } & \text { Reference mean fluorescence value (see [47]) } \\ \text { SDM } & \text { Second derivative maximum (see [42,48]) } \\ \text { stx1 } & \text { Shigatoxin 1 gene } \\ \text { Tm } & \text { Melting temperature }\end{array}$

\section{Appendix A. Melting Point Analysis of Borrelia Specific Probes}

Table A1. Pair-wise comparisons of the melting temperature of Borrelia. All combinations of melting points were subjected to the parametric pairwise multiple comparisons tests by means of the Tukey HSD test. All analyzes with a $p$ value less than 0.05 are shown.

\begin{tabular}{lrrrr}
\hline Comparison & Difference $\left({ }^{\circ} \mathbf{C}\right)$ & Lower & Upper & $p_{\text {adj }}$ \\
\hline B. bavariensis vs. B. afzelii & -1.55 & -1.98 & -1.12 & $<0.001$ \\
B. bissettii vs. B. afzelii & -2.70 & -3.13 & -2.27 & $<0.001$ \\
B. burgdorferi vs. B. afzelii & 0.46 & 0.02 & 0.89 & 0.03 \\
B. lusitaniae vs. B. afzelii & -3.48 & -3.91 & -3.04 & $<0.001$ \\
B. spielmanii vs. B. afzelii & -4.24 & -4.67 & -3.81 & $<0.001$ \\
B. bissettii vs. B. bavariensis & -1.15 & -1.58 & -0.72 & $<0.001$ \\
B. burgdorferi vs. B. bavariensis & 2.00 & 1.57 & 2.44 & $<0.001$ \\
B. garinii vs. B. bavariensis & 1.56 & 1.12 & 1.99 & $<0.001$ \\
B. kurtenbachii vs. B. bavariensis & 1.52 & 1.09 & 1.96 & $<0.001$ \\
B. lusitaniae vs. B. bavariensis & -1.93 & -2.36 & -1.50 & $<0.001$ \\
B. spielmanii vs. B. bavariensis & -2.69 & -3.12 & -2.26 & $<0.001$ \\
\hline
\end{tabular}


Table A1. Cont.

\begin{tabular}{lrrrr}
\hline Comparison & Difference $\left({ }^{\circ} \mathbf{C}\right)$ & Lower & Upper & $\mathbf{p}_{\text {adj }}$ \\
\hline B. valaisiana vs. B. bavariensis & 1.79 & 1.35 & 2.22 & $<0.001$ \\
B. burgdorferi vs. B. bissettii & 3.15 & 2.72 & 3.59 & $<0.001$ \\
B. garinii vs. B. bissettii & 2.71 & 2.27 & 3.14 & $<0.001$ \\
B. kurtenbachii vs. B. bissettii & 2.68 & 2.24 & 3.11 & $<0.001$ \\
B. lusitaniae vs. B. bissettii & -0.78 & -1.21 & -0.35 & $<0.001$ \\
B. spielmanii vs. B. bissettii & -1.54 & -1.97 & -1.11 & $<0.001$ \\
B. valaisiana vs. B. bissettii & 2.94 & 2.50 & 3.37 & $<0.001$ \\
B. garinii vs. B. burgdorferi & -0.45 & -0.88 & -0.01 & 0.04 \\
B. kurtenbachii vs. B. burgdorferi & -0.48 & -0.91 & -0.05 & 0.02 \\
B. lusitaniae vs. B. burgdorferi & -3.93 & -4.37 & -3.50 & $<0.001$ \\
B. spielmanii vs. B. burgdorferi & -4.69 & -5.13 & -4.26 & $<0.001$ \\
B. lusitaniae vs. B. garinii & -3.49 & -3.92 & -3.05 & $<0.001$ \\
B. spielmanii vs. B. garinii & -4.25 & -4.68 & -3.81 & $<0.001$ \\
B. lusitaniae vs. B. kurtenbachii & -3.45 & -3.89 & -3.02 & $<0.001$ \\
B. spielmanii vs. B. kurtenbachii & -4.22 & -4.65 & -3.78 & $<0.001$ \\
B. spielmanii vs. B. lusitaniae & -0.76 & -1.20 & -0.33 & $<0.001$ \\
B. valaisiana vs. B. lusitaniae & 3.71 & 3.28 & 4.15 & $<0.001$ \\
B. valaisiana vs. B. spielmanii & 4.48 & 4.04 & 4.91 & $<0.001$ \\
\hline
\end{tabular}

\section{Appendix B. Duplex LoopTag qPCR on a Planar Microbead Assays}

Aim: Planar microbead assays are an alternative to microarrays and suspension arrays since both enable the simultaneous monitoring of multiple targets in a single test. Different probe systems on microbead surfaces have been proposed [24]. We describe a qPCR approach based on microbeads, in which multiplexing is based on the number of microbead populations. The details on this coding principle are described in [24,47]. This is interesting for diagnostics, since assays with a high multiplex can in principle be built up. In our case study, an application of the LoopTag probe system is shown on a planar microbead assay. Figure A1 explains the concept of the LoopTag microbead probe system.

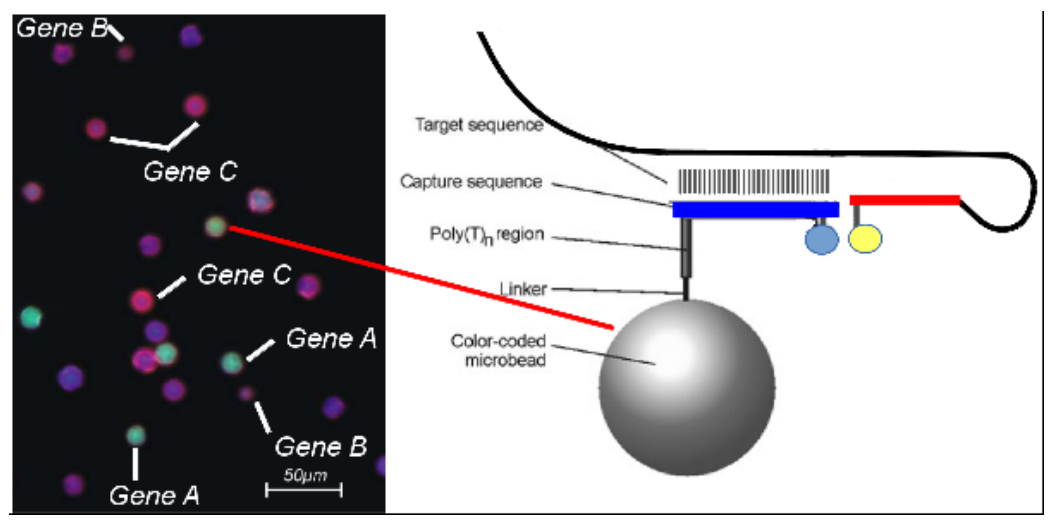

Figure A1. LoopTag probe system microbead real-time PCR. LoopTag formation. Right: Target specific LoopTag probes are assigned to a microbead population, which can be distinguished by its encoding dyes and size. In this cartoon three different populations encode the probe A (negative control), and the target sequences B (stx1) and C (estA1). Left: The target biomolecules are detected on the microbead surface as red corona. The LoopTag primer (red) labels the amplicon with a dye, in this case the quencher BHQ2 (yellow dot). One part of the LoopTag amplicon hybridizes to the microbead bound LoopTag capture probe (blue). The capture probe is labeled with a dye, in this case the fluorophor Atto647N (blue dot). The FRET signal is monitored in real-time during the annealing and elongation phase. Hence, hybridization analysis and differentiation by melting curve analysis are possible. 
Methods and Materials: To keep this supplement concise we refer to a summary about our VideoScan platform in the study [47]. In short, it is a fully automated microscopy system that allows cells, solutions, microbead assays, or combinations thereof to be analyzed by means of bioimage informatics. Measurements can be carried out in qPCR format using the VideoScan platform.

In the Borrelia study we used E. coli as control. Since Borrelia sample material is precious, we decided to conduct the study with DNA material from E. coli from our strain collection. We picked two representative diagnostically relevant genes commonly associated with Shigatoxin-producing E. coli (STEC; Shigatoxin 1 gene stx1) and enterotoxigenic E. coli (ETEC; heat-stable toxin I gene estA1).

- $\quad$ Base primer sequences were taken from [49] and transformed into a LoopTag probe system as described above. An important difference is that a part of the probes are bound to the surface of microbeads, so that they can function as catcher molecules (Table A2). The gene estA1 (length of amplification product: $158 \mathrm{bp}$ ) and stx1 (length of amplification product: $211 \mathrm{bp}$ ) were used in this proof-of-concept study.

- Colonies of E. coli strains containing genes estA1 and stx1 were inoculated in $1.5 \mathrm{~mL}$ LB medium. After incubation at $37^{\circ} \mathrm{C}$ overnight cells were separated, re-suspended in $300 \mu \mathrm{L}$ of water, and lysed by heating at $99^{\circ} \mathrm{C}$ for $10 \mathrm{~min}$. Cell debris were removed by centrifugation and supernatant was stored at $-20^{\circ} \mathrm{C}$. Thawed lysates were directly used as templates for PCR.

Table A2. Primer and probes for the microbead assay. Labels: Atto647N; BHQ2, Black Hole Quencher 2; 2B, dual biotin. Melting temperatures (Tm) were calculated with PerlPrimer.

\begin{tabular}{ll}
\hline Function & Sequence \\
\hline est $A 1 \mathrm{fw}$ & BHQ2-ATCTACCAACTGAATCACTTGACTCTT (GC: $\left.37 \%, 27 \mathrm{nt}, \mathrm{Tm}: 62.45^{\circ} \mathrm{C}\right)$ \\
est $A 1 \mathrm{rv}$ & TTAATAACATCCAGCACAGG (GC: $\left.40 \%, 20 \mathrm{nt}, \mathrm{Tm}: 55.57{ }^{\circ} \mathrm{C}\right)$ \\
est $1 \mathrm{p}$ probe & 2B-AGTCTCTAATGTAATTTCTCTTTTGTAGAT-Atto647N $\left(\mathrm{GC}: 28 \%, 32 \mathrm{nt}, \mathrm{Tm}: 61.51{ }^{\circ} \mathrm{C}\right)$ \\
st $1 \mathrm{fw}$ & BHQ2-ATGTATGTTGCAGGGATCAGTCGT $\left(\mathrm{GC}: 45 \%, 24 \mathrm{nt}, \mathrm{Tm}: 64.57^{\circ} \mathrm{C}\right)$ \\
st $x 1 \mathrm{rv}$ & AGAACGCCCACTGAGATCATC $\left(\mathrm{GC}: 52 \%, 21 \mathrm{nt}, \mathrm{Tm}: 62.41^{\circ} \mathrm{C}\right)$ \\
st $x 1$ probe & 2B-GTCAACGAATGGCGATTTATCTGCATCCCGTACAT-Atto647N $\left(\mathrm{GC}: 45 \%, 35 \mathrm{nt}, \mathrm{Tm}: 62.41{ }^{\circ} \mathrm{C}\right)$ \\
\hline
\end{tabular}

Carboxylated PMMA microbeads were coated with streptavidin as described in [47] and subjected to a qPCR reaction and melting curve analysis in the VideoScan platform.

1. Briefly, 300,000 microbeads were re-suspended in $100 \mu \mathrm{L}$ of $100 \mathrm{mM}$ methylimidazole (MeIm, Sigma, Milwaukee, WI, USA) buffer ( $\mathrm{pH} 7.0$ ) containing $25 \mathrm{mg} / \mathrm{mL}$ $N$-(3-dimethy-laminopropyl)- $N^{\prime}$-ethylcarbodiimide hydrochloride (EDC, Sigma). Activated microbeads were incubated with streptavidin solution $(300 \mu \mathrm{g} / \mathrm{mL}$ in MeImbuffer for $5 \mathrm{~h}$ at $50{ }^{\circ} \mathrm{C}$ ) with continuous agitation for covalent cross-linking. After washing three times with TBST buffer, streptavidin-coated microbeads were ready for loading with biotinylated oligonucleotides. Microbeads were mixed with $100 \mu \mathrm{L}$ TBST buffer containing $100 \mathrm{nM}$ of 5'-bis-biotinylated and 3'-Atto647N-labeled oligonucleotides (biomers.net, Ulm, Germany). After $15 \mathrm{~min}$ incubation at room temperature, unbound oligonucleotides were removed by washing microbeads three times with $200 \mu \mathrm{L}$ TBST. Finally, microbeads were exposed to $95^{\circ} \mathrm{C}$ for $10 \mathrm{~min}$.

2. All PCR reactions were conducted within a volume of $20 \mu \mathrm{L}$ Biotherm polymerase buffer (Genecraft, Colone, Germany) containing $5 \mathrm{mM} \mathrm{MgCl}, 200 \mu \mathrm{M}$ of each dNTP, $250 \mu \mathrm{M}$ of BHQ2 labeled fw primer, $250 \mu \mathrm{M}$ of rv primer, 20-200 streptavidincoupled microbeads with bound bis-biotinylated capture probes, 1 U Biotherm DNApolymerase (Genecraft), and $1 \mu \mathrm{L}$ of $E$. coli lysate as template. The mixture was transferred into a cavity of a nucleolink TopYield strip (Nunc, Roskilde, Denmark), covered with $30 \mu \mathrm{L}$ of mineral oil, and the strip was placed into sockets of the heating and cooling unit. Here, a PCR was performed using the following program: $4 \mathrm{~min}$, $94{ }^{\circ} \mathrm{C}, 40 \times\left(60 \mathrm{~s} 94{ }^{\circ} \mathrm{C}, 60 \mathrm{~s} 55{ }^{\circ} \mathrm{C}, 90 \mathrm{~s} 72{ }^{\circ} \mathrm{C}\right), 5 \mathrm{~min} 72^{\circ} \mathrm{C}$. We monitored microbead 
surface fluorescence while the temperature was increased at $1{ }^{\circ} \mathrm{C} / \mathrm{min}$ starting from $35^{\circ} \mathrm{C}$ to $90^{\circ} \mathrm{C}$. By use of the VideoScan technology fluorescence images of microbeads on the bottom of the well were taken after every cycle $\left(55^{\circ} \mathrm{C}\right)$. Images were evaluated with imaging processing software allowing the recognition of microbeads, the assignment to a population and the determination of their surface fluorescence.

3. The mean fluorescence value for every population (rMFI $\rightarrow$ RFU) was plotted against the cycle number leading to the shown real-time kinetic curve. The amplification curve data were pre-processed and analyzed using the $q p c R$ (v. 1.4.1) [50], MBmca, and chipPCR packages. After finishing real-time duplex PCR, a melting curve analysis was done as described in $[39,43]$.

Results and Conclusions:

We observed a curve shape, which resembles the typical sigmoid shape of quantitative PCRs (Figure 2). Amplification curves were fitted with a Richardson function. The Cq values were determined by the second derivative maximum method. The stx 1 amplification curve had a $\mathrm{Cq}$ value of 20.4, and the est $A 1$ amplification curve had a $\mathrm{Cq}$ value of 18.6 (Figure A2A). Next we performed a melting curve analysis on the surfaces of microbeads. The curve for $s t x 1$ had a Tm value of $63.2^{\circ} \mathrm{C}$ and for est $A 1$ a Tm value of $53.9^{\circ} \mathrm{C}$ (Figure A2B). Negative controls were not amplified. In this proof-of-concept study the LoopTag probe system was easily applicable to a planar microbead-based assay.
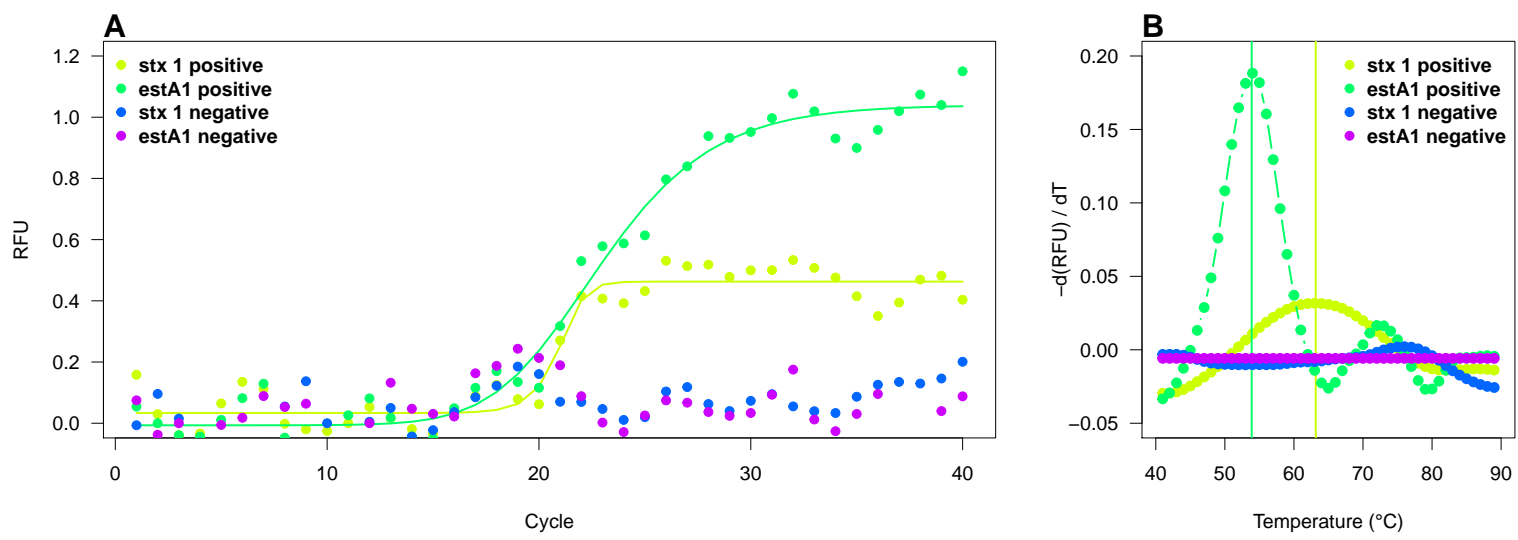

Figure A2. Quantitative PCR and melting curve analysis on the surface of microbeads. (A) Amplification of E. coli genes stx1 nd estA1 was monitored in a duplex LoopTag microbead assay. Negative controls (water control) were not amplified. (B) The melting temperature on the surface was determined for both genes. st $x 1$ and est 11 had different melting temperatures of $63.2^{\circ} \mathrm{C}$ and $53.9^{\circ} \mathrm{C}$, respectively.

Our measuring system was not further optimized for measuring the LoopTag system on microbeads. For example, we can observe a periodicity (wave-shaped amplification curve) as was also observed for commercial systems [51]. Though the signal-to-noise ratio is not optimal, this also led to the signal differing significantly from the background.

The evaluation and analysis was carried out in our case using the VideoScan platform. The principle should also be applicable to other planar assays. For the analysis of the image data, we use the proprietary FastFluoScan software (Attomol GmbH, Germany). However, other open source bioimage informatics software (as reviewed in [52]) could also be used for such scientific questions.

\section{References}

1. Hildebrandt, A.; Schmidt, K.H.; Wilske, B.; Dorn, W.; Straube, E.; Fingerle, V. Prevalence of four species of Borrelia burgdorferi sensu lato and coinfection with Anaplasma phagocytophila in Ixodes ricinus ticks in central Germany. Eur. J. Clin. Microbiol. Infect. Dis. Off. Publ. Eur. Soc. Clin. Microbiol. 2003, 22, 364-367. [CrossRef] [PubMed]

2. Lee, S.H.; Lee, J.H.; Park, H.S.; Jang, W.J.; Koh, S.E.; Yang, Y.M.; Kim, B.J.; Kook, Y.H.; Park, K.H. Differentiation of Borrelia burgdorferi sensu lato through groEL gene analysis. FEMS Microbiol. Lett. 2003, 222, 51-57. [CrossRef] 
3. Knoll, S.; Springer, A.; Hauck, D.; Schunack, B.; Pachnicke, S.; Fingerle, V.; Strube, C. Distribution of Borrelia burgdorferi s.l. and Borrelia miyamotoi in Ixodes tick populations in Northern Germany, co-infections with Rickettsiales and assessment of potential influencing factors. Med. Vet. Entomol. 2021. [CrossRef] [PubMed]

4. Tappe, J.; Jordan, D.; Janecek, E.; Fingerle, V.; Strube, C. Revisited: Borrelia burgdorferi sensu lato infections in hard ticks (Ixodes ricinus) in the city of Hanover (Germany). Parasites Vectors 2014, 7, 441. [CrossRef]

5. Răileanu, C.; Tauchmann, O.; Vasić, A.; Wöhnke, E.; Silaghi, C. Borrelia miyamotoi and Borrelia burgdorferi (sensu lato) identification and survey of tick-borne encephalitis virus in ticks from north-eastern Germany. Parasites Vectors 2020, 13, 106. [CrossRef]

6. Mendoza-Roldan, J.A.; Colella, V.; Lia, R.P.; Nguyen, V.L.; Barros-Battesti, D.M.; Iatta, R.; Dantas-Torres, F.; Otranto, D. Borrelia burgdorferi (sensu lato) in ectoparasites and reptiles in southern Italy. Parasites Vectors 2019, 12, 35. [CrossRef]

7. Hildebrandt, A.; Schmidt, K.H.; Fingerle, V.; Wilske, B.; Straube, E. Prevalence of granulocytic Ehrlichiae in Ixodes ricinus ticks in Middle Germany (Thuringia) detected by PCR and sequencing of a 16S ribosomal DNA fragment. FEMS Microbiol. Lett. 2002, 211, 225-230. [CrossRef]

8. Deutschmann, C.; Roggenbuck, D.; Schierack, P.; Rödiger, S. Autoantibody testing by enzyme-linked immunosorbent assay-A case in which the solid phase decides on success and failure. Heliyon 2020, 6, e03270. [CrossRef]

9. Lager, M.; Faller, M.; Wilhelmsson, P.; Kjelland, V.; Andreassen, A.; Dargis, R.; Quarsten, H.; Dessau, R.; Fingerle, V.; Margos, G.; et al. Molecular detection of Borrelia burgdorferi sensu lato-An analytical comparison of real-time PCR protocols from five different Scandinavian laboratories. PLoS ONE 2017, 12, e0185434. [CrossRef]

10. Primus, S.; Akoolo, L.; Schlachter, S.; Gedroic, K.; Rojtman, A.D.; Parveen, N. Efficient detection of symptomatic and asymptomatic patient samples for Babesia microti and Borrelia burgdorferi infection by multiplex qPCR. PLoS ONE 2018, 13, e0196748. [CrossRef]

11. Venczel, R.; Knoke, L.; Pavlovic, M.; Dzaferovic, E.; Vaculova, T.; Silaghi, C.; Overzier, E.; Konrad, R.; Kolenčík, S.; Derdakova, M.; et al. A novel duplex real-time PCR permits simultaneous detection and differentiation of Borrelia miyamotoi and Borrelia burgdorferi sensu lato. Infection 2016, 44, 47-55. [CrossRef] [PubMed]

12. Alby, K.; Capraro, G.A. Alternatives to Serologic Testing for Diagnosis of Lyme Disease. Clin. Lab. Med. 2015, 35, 815-825. [CrossRef] [PubMed]

13. Pabinger, S.; Rödiger, S.; Kriegner, A.; Vierlinger, K.; Weinhäusel, A. A survey of tools for the analysis of quantitative PCR (qPCR) data. Biomol. Detect. Quantif. 2014, 1, 23-33. [CrossRef]

14. Maurin, M. Real-time PCR as a diagnostic tool for bacterial diseases. Expert Rev. Mol. Diagn. 2012, 12, 731-754. [CrossRef]

15. Saiki, R.K.; Bugawan, T.L.; Horn, G.T.; Mullis, K.B.; Erlich, H.A. Analysis of enzymatically amplified beta-globin and HLA-DQ alpha DNA with allele-specific oligonucleotide probes. Nature 1986, 324, 163-166. [CrossRef] [PubMed]

16. Schmidt, B.L. PCR in laboratory diagnosis of human Borrelia burgdorferi infections. Clin. Microbiol. Rev. 1997, 10, 185-201. [CrossRef]

17. Holland, P.M.; Abramson, R.D.; Watson, R.; Gelfand, D.H. Detection of specific polymerase chain reaction product by utilizing the $5^{\prime}-3^{\prime}$ exonuclease activity of Thermus aquaticus DNA polymerase. Proc. Natl. Acad. Sci. USA 1991, 88, 7276-7280. [CrossRef] [PubMed]

18. Higuchi, R.; Fockler, C.; Dollinger, G.; Watson, R. Kinetic PCR Analysis: Real-time Monitoring of DNA Amplification Reactions. Nat. Biotechnol. 1993, 11, 1026-1030. [CrossRef]

19. Ruijter, J.M.; Lorenz, P.; Tuomi, J.M.; Hecker, M.; Hoff, M.J.B.V.D. Fluorescent-increase kinetics of different fluorescent reporters used for qPCR depend on monitoring chemistry, targeted sequence, type of DNA input and PCR efficiency. Microchim. Acta 2014, 181, 1689-1696. [CrossRef]

20. Forootan, A.; Sjöback, R.; Björkman, J.; Sjögreen, B.; Linz, L.; Kubista, M. Methods to determine limit of detection and limit of quantification in quantitative real-time PCR (qPCR). Biomol. Detect. Quantif. 2017, 12, 1-6. [CrossRef]

21. Navarro, E.; Serrano-Heras, G.; Castano, M.J.; Solera, J. Real-time PCR detection chemistry. Clin. Chim. Acta 2015, 439, 231-250. [CrossRef]

22. Kralik, P.; Ricchi, M. A Basic Guide to Real Time PCR in Microbial Diagnostics: Definitions, Parameters, and Everything. Front. Microbiol. 2017, 8, 108. [CrossRef]

23. Thelwell, N.; Millington, S.; Solinas, A.; Booth, J.; Brown, T. Mode of action and application of Scorpion primers to mutation detection. Nucleic Acids Res. 2000, 28, 3752-3761. [CrossRef]

24. Rödiger, S.; Liebsch, C.; Schmidt, C.; Lehmann, W.; Resch-Genger, U.; Schedler, U.; Schierack, P. Nucleic acid detection based on the use of microbeads: A review. Microchim. Acta 2014, 181, 1151-1168. [CrossRef]

25. Ranasinghe, R.T.; Brown, T. Fluorescence based strategies for genetic analysis. Chem. Commun. (Camb. Engl.) 2005, 44, 5487-5502. [CrossRef]

26. Bernard, P.S.; Ajioka, R.S.; Kushner, J.P.; Wittwer, C.T. Homogeneous multiplex genotyping of hemochromatosis mutations with fluorescent hybridization probes. Am. J. Pathol. 1998, 153, 1055-1061. [CrossRef]

27. Lay, M.J.; Wittwer, C.T. Real-time fluorescence genotyping of factor V Leiden during rapid-cycle PCR. Clin. Chem. 1997, 43, 2262-2267. [CrossRef] [PubMed]

28. Marras, S.A.; Kramer, F.R.; Tyagi, S. Multiplex detection of single-nucleotide variations using molecular beacons. Genet. Anal. Biomol. Eng. 1999, 14, 151-156. [CrossRef]

29. Schwaiger, M.; Péter, O.; Cassinotti, P. Routine diagnosis of Borrelia burgdorferi (sensu lato) infections using a real-time PCR assay. Clin. Microbiol. Infect. Off. Publ. Eur. Soc. Clin. Microbiol. Infect. Dis. 2001, 7, 461-469. [CrossRef] 
30. Lehmann, W.; Hanschmann, H.; Syring, M. Method and Probe/Primer System for the “Real Time" Detection of a Nucleic Acid Target. International Patent WO2008152144A1, 13 June 2008.

31. Bustin, S.; Huggett, J. qPCR primer design revisited. Biomol. Detect. Quantif. 2017, 14, 19-28. [CrossRef]

32. Zuker, M. Mfold web server for nucleic acid folding and hybridization prediction. Nucleic Acids Res. 2003, 31, 3406-3415. [CrossRef]

33. Marshall, O. Graphical Design of Primers with PerlPrimer. In PCR Primer Design; Yuryev, A., Ed.; Number 402 in Methods in Molecular Biology ${ }^{\mathrm{TM}}$; Humana Press: Totowa, NJ, USA, 2007; pp. 403-414. [CrossRef]

34. Ponti, G.; Maccaferri, M.; Manfredini, M.; Kaleci, S.; Mandrioli, M.; Pellacani, G.; Ozben, T.; Depenni, R.; Bianchi, G.; Pirola, G.M.; et al. The value of fluorimetry (Qubit) and spectrophotometry (NanoDrop) in the quantification of cell-free DNA (cfDNA) in malignant melanoma and prostate cancer patients. Clin. Chim. Acta 2018, 479, 14-19. [CrossRef]

35. Wright, K.; de Silva, K.; Purdie, A.C.; Plain, K.M. Comparison of methods for miRNA isolation and quantification from ovine plasma. Sci. Rep. 2020, 10, 825. [CrossRef]

36. Nakayama, Y.; Yamaguchi, H.; Einaga, N.; Esumi, M. Pitfalls of DNA Quantification Using DNA-Binding Fluorescent Dyes and Suggested Solutions. PLoS ONE 2016, 11, e0150528. [CrossRef] [PubMed]

37. Mao, F.; Leung, W.Y.; Xin, X. Characterization of EvaGreen and the implication of its physicochemical properties for qPCR applications. BMC Biotechnol. 2007, 7, 76. [CrossRef]

38. Rödiger, S.; Friedrichsmeier, T.; Kapat, P.; Michalke, M. RKWard: A comprehensive graphical user interface and integrated development environment for statistical analysis with R. J. Stat. Softw. 2012, 49, 1-34. [CrossRef]

39. Rödiger, S.; Burdukiewicz, M.; Blagodatskikh, K.A.; Schierack, P. R as an Environment for the Reproducible Analysis of DNA Amplification Experiments. R J. 2015, 7, 127-150. [CrossRef]

40. Makowski, D.; Ben-Shachar, M.S.; Patil, I.; Lüdecke, D. Automated Results Reporting as a Practical Tool to Improve Reproducibility and Methodological Best Practices Adoption; Technical Report; Easystats: Rocklin, CA, USA, 2021.

41. Rödiger, S.; Burdukiewicz, M.; Schierack, P. chipPCR: An R package to pre-process raw data of amplification curves. Bioinformatics 2015, 31, 2900-2902. [CrossRef] [PubMed]

42. Spiess, A.N.; Deutschmann, C.; Burdukiewicz, M.; Himmelreich, R.; Klat, K.; Schierack, P.; Rödiger, S. Impact of Smoothing on Parameter Estimation in Quantitative DNA Amplification Experiments. Clin. Chem. 2015, 61, 379-388. [CrossRef]

43. Rödiger, S.; Böhm, A.; Schimke, I. Surface Melting Curve Analysis with R. R J. 2013, 5, 37-53. [CrossRef]

44. Aguero-Rosenfeld, M.E.; Wang, G.; Schwartz, I.; Wormser, G.P. Diagnosis of Lyme Borreliosis. Clin. Microbiol. Rev. 2005, 18, 484-509. [CrossRef] [PubMed]

45. Hoffmann, A.; Müller, T.; Fingerle, V.; Noll, M. Presence of Human Pathogens of the Borrelia burgdorferi sensu lato Complex Shifts the Sequence Read Abundances of Tick Microbiomes in Two German Locations. Microorganisms 2021, 9, 1814. [CrossRef]

46. Burdukiewicz, M.; Spiess, A.N.; Blagodatskikh, K.A.; Lehmann, W.; Schierack, P.; Rödiger, S. Algorithms for automated detection of hook effect-bearing amplification curves. Biomol. Detect. Quantif. 2018, 16, 1-4. [CrossRef] [PubMed]

47. Rödiger, S.; Schierack, P.; Böhm, A.; Nitschke, J.; Berger, I.; Frömmel, U.; Schmidt, C.; Ruhland, M.; Schimke, I.; Roggenbuck, D.; et al. A highly versatile microscope imaging technology platform for the multiplex real-time detection of biomolecules and autoimmune antibodies. Adv. Biochem. Eng. 2013, 133, 35-74. [CrossRef]

48. Ruiz-Villalba, A.; Ruijter, J.M.; van den Hoff, M.J.B. Use and Misuse of Cq in qPCR Data Analysis and Reporting. Life 2021, 11, 496. [CrossRef]

49. Frömmel, U.; Lehmann, W.; Rödiger, S.; Böhm, A.; Nitschke, J.; Weinreich, J.; Groß, J.; Roggenbuck, D.; Zinke, O.; Ansorge, H.; et al. Adhesion of Human and Animal Escherichia coli Strains in Association with Their Virulence-Associated Genes and Phylogenetic Origins. Appl. Environ. Microbiol. 2013, 79, 5814-5829. [CrossRef]

50. Ritz, C.; Spiess, A.N. qpcR: An R package for sigmoidal model selection in quantitative real-time polymerase chain reaction analysis. Bioinformatics 2008, 24, 1549-1551. [CrossRef] [PubMed]

51. Spiess, A.N.; Rödiger, S.; Burdukiewicz, M.; Volksdorf, T.; Tellinghuisen, J. System-specific periodicity in quantitative real-time polymerase chain reaction data questions threshold-based quantitation. Sci. Rep. 2016, 6, 38951. [CrossRef]

52. Schneider, J.; Weiss, R.; Ruhe, M.; Jung, T.; Roggenbuck, D.; Stohwasser, R.; Schierack, P.; Rödiger, S. Open source bioimage informatics tools for the analysis of DNA damage and associated biomarkers. J. Lab. Precis. Med. 2019, 4, 1-27. [CrossRef] 